\title{
Spontaneous Formation of Gold Nanoparticles on Graphene by Galvanic Reaction through Graphene
}

\author{
Yohwan Park, Jin Young Koo, Soyoung Kim, ${ }^{\circledR}$ and Hee Cheul Choi*(-) \\ Department of Chemistry, Pohang University of Science and Technology (POSTECH), Pohang 37673, Republic of Korea
}

Supporting Information

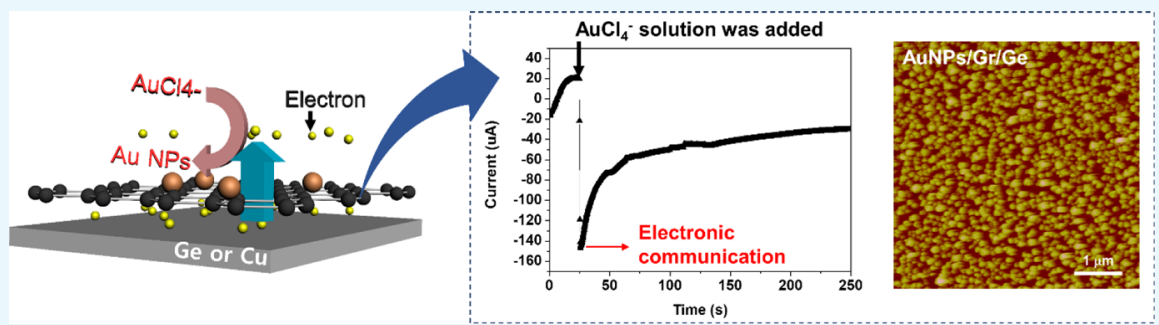

ABSTRACT: We demonstrate an effective and facile method for the deposition of gold nanoparticles (AuNPs) on graphene by using spontaneous galvanic reaction. Despite the interest and importance of the hybrid structure of noble metal-deposited graphene has been considerably increased for its fundamental knowledge in chemical and physical sciences and for its various applications, the progress of this subject is very slow mainly because of the lack of synthetic methods for such structures, especially that are not free from chemical contamination and usage of complex and expensive equipment. Therefore, we developed a new method allowing chemically pure AuNPs/graphene hybrid structures employing galvanic reaction. The spontaneous galvanic reaction was derived from reductant/graphene/oxidant sandwich structures, such as Au ions/graphene/ $\mathrm{Ge}$ wafer and $\mathrm{Au}$ ions/graphene/copper foil, by placing Au ion solution droplets on graphene transferred on a germanium wafer or as made graphene on $\mathrm{Cu}$ foil, respectively. According to scanning electron microscopy and atomic force microscopy results, it was confirmed that AuNPs were successfully formed on the graphene surface. This result implies two important points. One is that the formation of pure AuNPs on graphene is possible without using other chemicals frequently required for conventional NP preparation. The other one is that it was experimentally demonstrated that there are electronic communications between the oxidant and reductant that are separated by graphene, through which electrons can pass freely.

\section{INTRODUCTION}

The hybrid structures of metal nanoparticles (NPs) deposited on graphene have attracted a great deal of attention because of their potential applications in various fields in physics, ${ }^{1}$ sensors, ${ }^{2}$ catalysts, ${ }^{3}$ and so forth. Therefore, many researchers have contributed to develop methods to construct such hybrid structures by using external potential, ${ }^{4}$ laser, ${ }^{5}$ various additives, $^{6}$ and so forth. However, most of the proposed methods have various limitations including cost ineffectiveness and process complexity because they require the use of many chemicals, additional equipment, or external energy source to apply. Among various electrochemical deposition processes, spontaneous galvanic reaction has been considered as one of the simplest and facile reactions that can form metal NPs when the metal source ions are brought into contact with the target substrate if their redox potential values are well matched. Previously, galvanic reaction was successfully employed to spontaneously form noble metal NPs, such as $\mathrm{Au}, \mathrm{Pt}$, and $\mathrm{Pd}$ NPs, on less noble metal or semiconductor substrates such as $\mathrm{Cu}$ and $\mathrm{Ge}$ without additives such as reducing agents, $\mathrm{pH}$ adjusters, and complexing agents. ${ }^{8}$ When the noble metal cations and the substrate are in contact, the electrons withdrawn from the reductant substrate by oxidation are used to reduce the metal cations into the corresponding metal NPs on the substrate. In the p-type semiconducting singlewalled carbon nanotube case, metal deposition could also be achieved spontaneously and directly by the galvanic reaction. ${ }^{9}$ However, in the graphene case, the galvanic reaction could not be applied because of its intrinsic semimetallic electronic structure. To overcome this limitation, we designed reductant/ graphene/oxidant sandwich structures, so that the reductant and oxidant could cause the galvanic reaction and exchange charges through graphene and eventually form AuNPs on the surface of graphene.

\section{RESULTS AND DISCUSSION}

We chose germanium $(\mathrm{Ge})$ and copper $(\mathrm{Cu})$ as reductants that would reduce noble metal cations $\left(\mathrm{Au}^{3+}\right)$ upon contact into the corresponding AuNPs according to thermodynamically favorable redox potential energy (Table 1$).^{10}$

To achieve the spontaneous metal deposition on graphene, we first fabricated samples of the graphene/Ge structure by

Received: August 20, 2019

Accepted: October 14, 2019

Published: October 25, 2019 
Table 1. Electrochemical Redox Potential for Germanium/ Gold and Copper/Gold Systems Available for Galvanic Reactions

\begin{tabular}{clcc} 
system & \multicolumn{1}{c}{ redox couple } & $E^{\mathrm{o}}(\mathrm{V})$ & $E_{\text {cell }}^{\mathrm{o}}(\mathrm{V})$ \\
$\mathrm{Au} / \mathrm{Ge}$ & $\mathrm{AuCl}_{4}{ }^{-}+3 \mathrm{e}^{-} \rightarrow \mathrm{Au}+4 \mathrm{Cl}^{-}$ & +1.002 & +0.878 \\
& $\mathrm{Ge} \rightarrow \mathrm{Ge}^{4+}+4 \mathrm{e}^{-}$ & -0.124 & \\
$\mathrm{Au} / \mathrm{Cu}$ & $\mathrm{AuCl}_{4}{ }^{-}+3 \mathrm{e}^{-} \rightarrow \mathrm{Au}+4 \mathrm{Cl}^{-}$ & +1.002 & +0.660 \\
& $\mathrm{Cu} \rightarrow \mathrm{Cu}^{2+}+2 \mathrm{e}^{-}$ & -0.342 & \\
\hline
\end{tabular}

transferring graphene that was separately synthesized by the chemical vapor deposition (CVD) process onto a Ge wafer substrate. (Detailed experimental method is described in the Method Section). After that, an aliquot of aqueous solution of $\mathrm{AuCl}_{4}^{-}(0.1 \mathrm{M})$ was dropped on graphene/Ge for $5 \mathrm{~min}$ (Scheme 1a). As a result, spherical AuNPs in a quite uniform

Scheme 1. (a) Experimental Design To Deposit AuNPs on Graphene as the Substrate ( $\mathrm{Ge}$ or $\mathrm{Cu}$ )/Graphene/ $\mathrm{AuCl}_{4}{ }^{-}$ Sandwich Structure; (b) Scheme of Spontaneous AuNPs Deposition on Graphene Mechanism That Shows Charge Communication between the Substrate ( $\mathrm{Ge}$ or $\mathrm{Cu}$ ) and $\mathrm{AuCl}_{4}{ }^{-}$Ions through Graphene
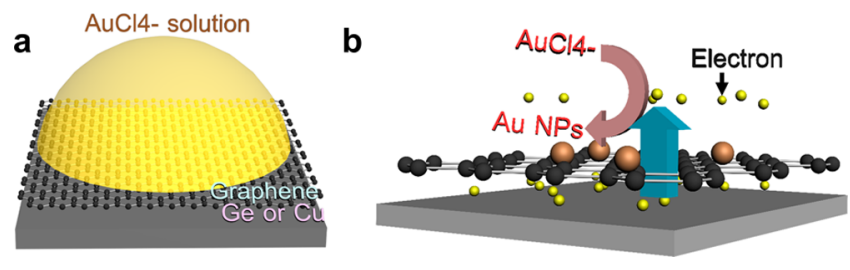

size $(80-100 \mathrm{~nm})$ were found on graphene as observed by optical microscopy, scanning electron microscopy (SEM), and atomic force microscopy (AFM) (Figures 1a,b and S1a). The size and uniformity of the resulting AuNP are largely affected by several reaction parameters including the concentration of $\mathrm{AuCl}_{4}{ }^{-}$, reaction time, and so forth. In addition, Raman spectra confirmed the successful transfer of graphene on the $\mathrm{Ge}$ substrate, and the formation of AuNPs was observed by enhanced Raman scattering because of AuNPs formed on the top of the graphene surface (Figure S2). The elemental composition of the AuNPs and substrate was investigated by X-ray photoelectron spectroscopy (XPS) (Figure S3).

To confirm the origin of the electrons from Ge rather than providing electrons from graphene itself, a series of control experiments were performed. We conducted the same reaction by using (1) the as-synthesized graphene on copper (graphene/Cu) instead of $\mathrm{Ge}$, which also has matched the thermodynamically favorable redox potential (Table 1) to $\mathrm{AuCl}_{4}^{-}$solution, and (2) the transferred graphene on $\mathrm{SiO}_{2}$ (graphene $/ \mathrm{SiO}_{2}$ ) to make sure that the origin of the electron is $\mathrm{Ge}$ or $\mathrm{Cu}$, rather than graphene itself, thus to assure that graphene acts only as a window for electrons. In the graphene/ $\mathrm{Cu}$ case, $\mathrm{Cu}$ foil has an advantage over $\mathrm{Ge}$ for preparing samples because $\mathrm{Cu}$ foil used for the growth of graphene by CVD still can be used without any extra treatment, which guarantees convenience and clean conditions. On the graphene $/ \mathrm{Cu}$ samples, spherical AuNPs of 50-80 nm size were formed into a film (Figures $1 \mathrm{c}, \mathrm{d}$ and $\mathrm{S} 1 \mathrm{~b}$ ). Also, we observed high crystallinity of the resulting AuNPs by highresolution transmission electron microscopy (HR-TEM) (Figure S4). On the other hand, in the case of graphene/ $\mathrm{SiO}_{2}$, no AuNP was found on graphene (Figure S5). This

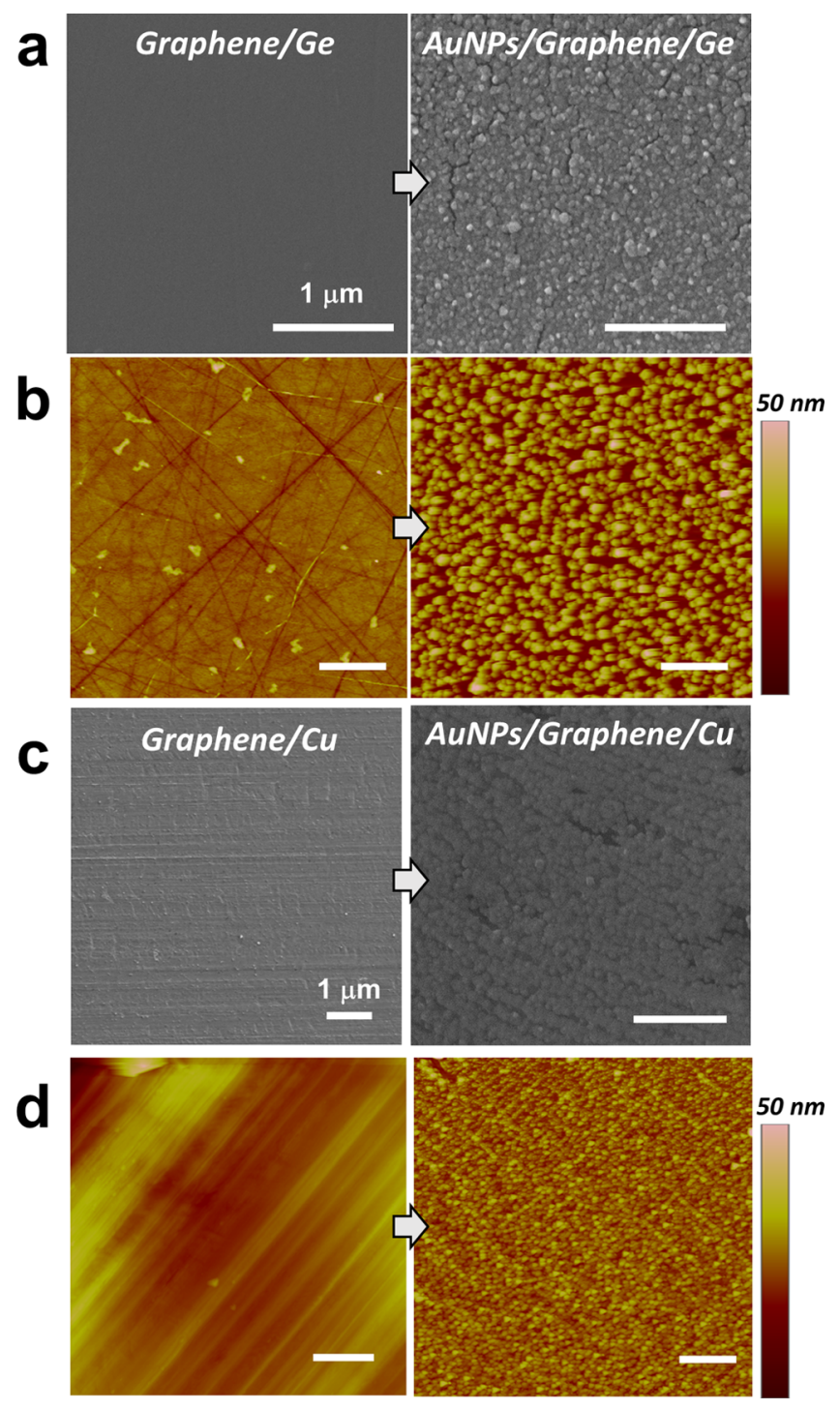

Figure 1. $(a-d) S E M$ and $A F M$ images of graphene/Ge and graphene/ $\mathrm{Cu}$ after dropping $\mathrm{AuCl}_{4}{ }^{-}$solution.

clarifies that graphene itself does not involve in the reduction of $\mathrm{AuCl}_{4}{ }^{-}$, so the electrons used for the formation of AuNPs on graphene $/ \mathrm{Ge}$ and graphene/ $\mathrm{Cu}$ must come from $\mathrm{Ge}$ and $\mathrm{Cu}$. In other words, the galvanic reaction that took place across the graphene could offer direct and facile metal NP deposition by using the electrons from $\mathrm{Ge}$ or $\mathrm{Cu}$ (Scheme $\mathrm{1b}$ ).

In general, the size of noble metal NPs formed by the spontaneous galvanic reaction is highly affected by the concentration of the oxidant solution. ${ }^{11}$ Therefore, we examined the change in AuNP size by varying the concentration of $\mathrm{AuCl}_{4}^{-}$solution (Figure 2a). The $\mathrm{AuCl}_{4}^{-}$ solutions at different concentrations of $0.5,1.0$, and $2.0 \mathrm{mg} /$ $\mathrm{mL}$ resulted in the size increase of AuNPs from 49-106 and $85-141$ to $289-690 \mathrm{~nm}$, respectively. The trend indicates that the amount of electrons withdrawn from the Ge substrate differs depending on the concentration of the $\mathrm{AuCl}_{4}^{-}$solution and it affects the size of the resulting AuNPs. Furthermore, to examine how many layers of graphene could be accessible for the spontaneous deposition by electrochemically generated electrons, the reaction was carried out with bilayer and trilayer graphene and exfoliated thick graphite. As a result, AuNPs grown on the bilayer graphene were similar to those of the 

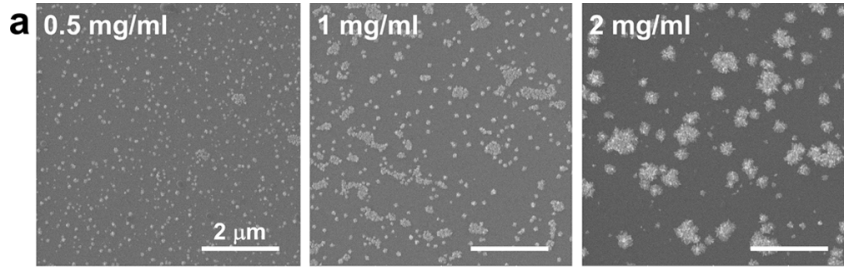

b
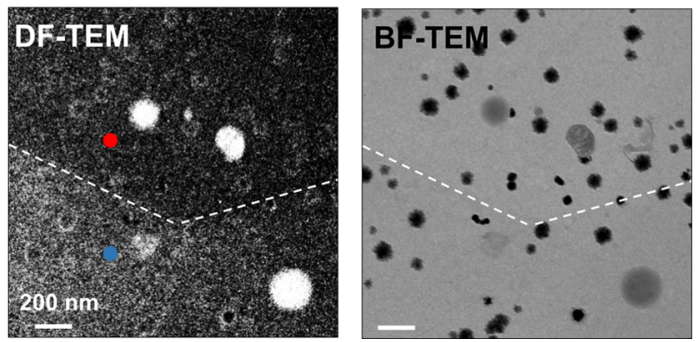

Figure 2. (a) SEM images of AuNPs which grown on graphene depending on the $\mathrm{AuCl}_{4}^{-}$concentration. (b) Dark-field (left) and bright-field (right) TEM images of the AuNPs/graphene structure. Red and blue dots show different grain, and the white dotted line shows grain boundary.

monolayer graphene, and slightly larger AuNPs were grown on the trilayer graphene (Figure S6), but no AuNP was formed on the exfoliated graphite on Ge (Figure S7). This indicates that charge communication between the reductant and oxidant is available for bilayer and trilayer graphene but not beyond them.

The chance for the formation of AuNPs in the gap space between the graphene layer and $\mathrm{Ge}$ or $\mathrm{Cu}$ substrates was excluded because it is well known that most of large ions and molecules including solvents are impermeable to graphene. ${ }^{12}$ Moreover, because all the reactions were performed by placing oxidant solution droplets on the top of graphene, there is no chance for the oxidant droplet to diffuse into the space between the graphene and substrate. Furthermore, to confirm whether AuNPs were formed on graphene grain or grain boundary, dark-field TEM measurement was conducted. As a result, we observed the area that shows different grains and grain boundaries (Figures $S 8$ and $2 \mathrm{~b}$ ) and confirmed that AuNPs were formed on the graphene crystal domain rather than on the grain boundary.

Meanwhile, another direct evidence of charge communication between the reductant and oxidant through graphene was obtained from the electrical current flow through graphene. For this, we designed an electrochemical cell to measure the electrical current generated upon the galvanic reaction through graphene to elucidate the accurate and direct observation of the charge communication through graphene (Figure 3a). The electrochemical cell has a hole with a specific area to compare the accurate amount of electrons, and $\mathrm{Ag} / \mathrm{AgCl}$ and $\mathrm{Pt}$ wire were used as the reference and counter electrode, respectively. (Detailed experimental method was described in Method section). When $\mathrm{AuCl}_{4}{ }^{-}$solution came into contact with $\mathrm{Cu}$ or graphene $/ \mathrm{Cu}$, the $\mathrm{Cu}$ was oxidized and AuNPs were formed. Because this means the electrons were extracted from $\mathrm{Cu}$, we measured the current as a function of time using electrochemical current measurements. Because the redox reaction occurs spontaneously and immediately, the initial current measurement was performed in deionized (DI) water, and the change was measured upon adding $\mathrm{AuCl}_{4}^{-}$solution while maintaining $0 \mathrm{~V}$ potential. To examine the tendency of
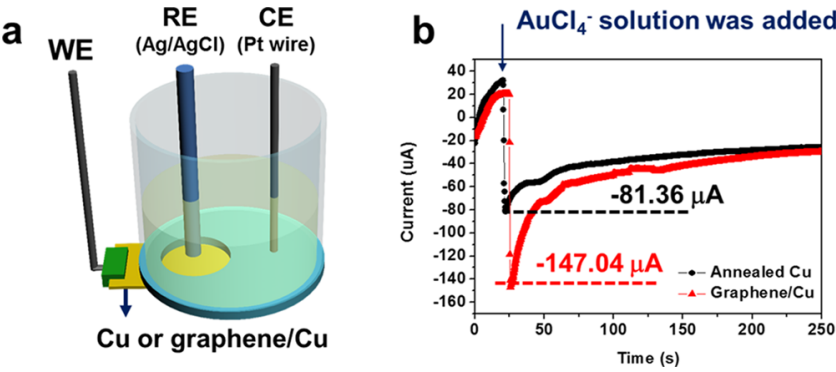

Figure 3. (a) Scheme of a lab-designed electrochemical cell for electrochemical current measurement. (WE: working electrode, RE: reference electrode, and CE: counter electrode). (b) Electrochemical current measurement of $\mathrm{Cu}$ and graphene/ $\mathrm{Cu}$ as a function of time while maintaining $0 \mathrm{~V}$ potential.

electron generation and the influence of the substrate on passing, a series of control experiments were performed. We conducted the electrochemical current measurements by using (1) $\mathrm{Cu}$ and (2) graphene/ $\mathrm{Cu}$. As a result, from $\mathrm{Cu}$ and graphene/ $\mathrm{Cu}$ cases, the instantaneous current increases to -81.36 and $-147.04 \mu \mathrm{A}$ were observed, respectively, and then a gradual decrease was observed (Figure $3 \mathrm{~b}$ ). The instantaneous current increase may owe to extracting electrons from $\mathrm{Cu}$, and the gradual decrease of the current indicates the accumulation of AuNPs. Also, the electrochemical current difference shown from $\mathrm{Cu}$ and graphene/Cu is believed to be originated from the native oxide layer present only on $\mathrm{Cu}$. The oxide-free graphene/ $\mathrm{Cu}$ shows a higher current level than the $\mathrm{Cu}$-containing oxide layer. Furthermore, when the bias voltage was increased to more negative, the current also increased, which means the negative bias voltage accelerates the oxidation of $\mathrm{Cu}$, allowing a higher current to flow (Figure S9). The concentration of $\mathrm{AuCl}_{4}^{-}$solution also influences the current flow because a high concentration of $\mathrm{AuCl}_{4}^{-}$drives higher mass transfer of $\mathrm{Cu}^{2+}$; hence, more electrons could be generated (Figure S10).

\section{CONCLUSIONS}

In conclusion, we demonstrated a direct and facile AuNPs deposition process on graphene by using galvanic reaction. An efficient electron transfer from the reductant to oxidant through graphene was induced by spontaneous galvanic redox reaction from reductant/graphene/oxidant sandwich structures and successfully resulted in AuNP deposition on graphene. It is also noteworthy that this result also confirmed the efficient charge communication through the graphene basal plane, by confirming that the charge transfer occurs through the graphene basal plane rather than through grain boundaries by performing TEM. Furthermore, the charge communication was proved by electrochemical current measurements that showed an immediate current increase. Our results have an importance as they not only suggest the simplest way for the fabrication of the metal NPs/graphene hybrid structure but also provide an opportunity to prove charge communication in a system divided by graphene, which can be further employed in various applications.

\section{METHOD SECTION}

General Information. All reagents were purchased from commercial sources and used without further purification. $\mathrm{HAuCl}_{4}$ (Sigma-Aldrich > 99.9 \%), Cu foil (Alfa Aesar, 0.025 mm thick, annealed, coated, $99.8 \%$, metals basis), and Ge(110) 
substrate (Crystal bank, $500 \mu \mathrm{m}$ thick, $\mathrm{N}$-type, undoped, $>50$ $\Omega \cdot \mathrm{cm}$ resistivity, single-side polished). All the SEM images were obtained by using a JEOL and AFM images using an atomic force microscope (Nanoscope III). Raman spectra were obtained using a confocal Raman spectrometer (WiTec), and XPS data were obtained at the Research Institute of Industrial Science and Technology (RIST, Korea) (VG ESCALAB 250, VG Scientific).

Synthesis of Graphene by CVD. A piece of $\mathrm{Cu}$ foil (Alfa Aesar, $0.025 \mathrm{~mm}$ thick, annealed, coated, 99.8\%, metal basis) was purified by soaking it in acetic acid (25\%) and dried by $\mathrm{N}_{2}$ (99.999\%) blowing. The $\mathrm{Cu}$ foil was located at the center of the $1 \mathrm{in}$. protection quartz tube inside a tube heating furnace under vacuum conditions $\left(\sim 4 \times 10^{-2}\right.$ Torr), and $\mathrm{H}_{2}$ gas (40 sccm, $\sim 12 \times 10^{-2}$ Torr) was flown for $10 \mathrm{~min}$ to flush the protection tube. After that, temperature was raised to $1050{ }^{\circ} \mathrm{C}$ and maintained for 10 minutes for annealing of $\mathrm{Cu}$ foil; then, the $\mathrm{CH}_{4}$ gas (purity, vendor) was supplied for $20 \mathrm{~min}$. Moreover, the $\mathrm{Cu}$ foil was fast cooled to $25^{\circ} \mathrm{C}$ by opening the lid of the tube furnace, and $\mathrm{CH}_{4}$ gas was turned off at $800{ }^{\circ} \mathrm{C}$.

Transfer Graphene from $\mathrm{Cu}$ to the Arbitrary Substrate. To transfer the graphene on a Ge substrate or graphene/AuNPs on a $\mathrm{SiO}_{2}$ substrate, the poly(methyl methacrylate) (PMMA) solution (MicroChem Corp., 950 PMMA A5) was spin-coated on one side of the as-synthesized graphene or graphene/AuNPs on $\mathrm{Cu}$ foil and graphene on the other side was removed by using oxygen plasma (Femto Science, COVANCE-MPR). $\mathrm{Cu}$ was then etched by using ammonium persulfate aqueous solution and washed using DI water. The PMMA/graphene film was fished by a $\mathrm{Ge}(110)$ or $\mathrm{SiO}_{2}$ substrate and heated at $180{ }^{\circ} \mathrm{C}$ for $2 \mathrm{~h}$ in air. Finally, PMMA was removed by soaking in acetone and annealed under $\mathrm{Ar}$ and $\mathrm{H}_{2}$ flow for $1 \mathrm{~h}$.

Electrochemical Current Measurements. A piece of $\mathrm{Cu}$ foil was annealed at $1050{ }^{\circ} \mathrm{C}$ for $10 \mathrm{~min}$ to equate conditions with the graphene/Cu sample. The electrochemical current measurements were performed using a Versastat 3.0. To measure the accurate and comparable current value, the electrochemical cell was designed to have a hole, which has a specific area to measure from the same exposed area. $\mathrm{Ag} / \mathrm{AgCl}$ and $\mathrm{Pt}$ wire were used as the reference and counter electrode, respectively. The scan rate was 0.2 point per second, and the bias voltage was $0 \mathrm{~V}$ for $300 \mathrm{~s}$.

\section{ASSOCIATED CONTENT}

\section{S Supporting Information}

The Supporting Information is available free of charge on the ACS Publications website at DOI: 10.1021/acsomega.9b02691.

Bright-field and dark-field optical microscopy images; Raman spectra of graphene/Ge; XPS spectra; HR-TEM image of AuNPs; SEM images; electron diffraction patterns, electrochemical current measurements; and AFM image (PDF)

\section{AUTHOR INFORMATION}

\section{Corresponding Author}

*E-mail: choihc@postech.edu.

\section{ORCID}

Soyoung Kim: 0000-0001-6679-7057

Hee Cheul Choi: 0000-0003-1002-1262

\section{Notes}

The authors declare no competing financial interest.

\section{ACKNOWLEDGMENTS}

The authors acknowledge funding from the Veteran Researcher Grant (no. 2019R1A2C2004259) managed by the National Research Foundation of Korea (NRF) and Samsung Electronics. TEM (HR-FE-TEM-2200FS with Cs corrected TEM) measurement was performed at National Institute for Nanomaterials Technology (NINT), Pohang, Republic of Korea.

\section{ABBREVIATIONS}

AuNPs, gold nanoparticles; $\mathrm{Cu}$, copper; $\mathrm{Ge}$, germanium

\section{REFERENCES}

(1) (a) Abraham, S.; König, M.; Srivastava, S. K.; Kumar, V.; Walkenfort, B.; Srivastava, A. Carbon nanostructure (0-3 dimensional) supported isolated gold nanoparticles as an effective SERS substrate. Sens. Actuators, B 2018, 273, 455-465. (b) Ren, W.; Fang, Y.; Wang, E. A Binary Functional Substrate for Enrichment and Ultrasensitive SERS Spectroscopic Detection of Folic Acid Using Graphene Oxide/Ag Nanoparticle Hybrids. ACS Nano 2011, 5, $6425-6433$

(2) (a) Lightcap, I. V.; Kamat, P. V. Graphitic Design: Prospects of Graphene-Based Nanocomposites for Solar Energy Conversion, Storage, and Sensing. Acc. Chem. Res. 2013, 46, 2235-2243. (b) Gutés, A.; Hsia, B.; Sussman, A.; Mickelson, W.; Zettl, A.; Carraro, C.; Maboudian, R. Graphene decoration with metal nanoparticles: Towards easy integration for sensing applications. Nanoscale 2012, 4, 438.

(3) (a) Li, Y.; Fan, X.; Qi, J.; Ji, J.; Wang, S.; Zhang, G.; Zhang, F. Gold nanoparticles-graphene hybrids as active catalysts for Suzuki reaction. Mater. Res. Bull. 2010, 45, 1413-1418. (b) Chen, S.; Xiang, Y.; Peng, C.; Xu, W.; Banks, M. K.; Wu, R. Synthesis of a novel graphene-based gold nanocomposite using PVEIM-b-PNIPAM as a stabilizer and its thermosensitivity for the catalytic reduction of 4nitrophenol. Inorg. Chem. Front. 2019, 6, 903-913. (c) Zhang, M.; Lu, X.; Wang, H.-Y.; Liu, X.; Qin, Y.; Zhang, P.; Guo, Z.-X. Porous gold nanoparticle/graphene oxide composite as efficient catalysts for reduction of 4-nitrophenol. RSC Adv. 2016, 6, 35945-35951.

(4) Lomax, D. J.; Dryfe, R. A. W. Electrodeposition of Au on basal plane graphite and graphene. J. Electroanal. Chem. 2018, 819, 374383.

(5) Torres-Mendieta, R.; Ventura-Espinosa, D.; Sabater, S.; Lancis, J.; Mínguez-Vega, G.; Mata, J. A. In situ decoration of graphene sheets with gold nanoparticles synthetized by pulsed laser ablation in liquids. Sci. Rep. 2016, 6, 30478.

(6) (a) Liu, K.; Liu, L.; Luo, Y.; Jia, D. One-step synthesis of metal nanoparticle decorated graphene by liquid phase exfoliation. J. Mater. Chem. 2012, 22, 20342. (b) Zhuo, Q.; Ma, Y.; Gao, J.; Zhang, P.; Xia, Y.; Tian, Y.; Sun, X.; Zhong, J.; Sun, X. Facile Synthesis of Graphene/ Metal Nanoparticle Composites via Self-Catalysis Reduction at Room Temperature. Inorg. Chem. 2013, 52, 3141-3147. (c) Ioni, Y.; Buslaeva, E.; Gubin, S. Synthesis of Graphene with Noble Metals Nanoparticles on its Surface. Mater. Today: Proc. 2016, 3, S209-S213. (d) Jasuja, K.; Berry, V. Implantation and Growth of Dendritic Gold Nanostructures on Graphene Derivatives: Electrical Property Tailoring and Raman Enhancement. ACS Nano 2009, 3, 23582366. (e) Xu, C.; Wang, X.; Zhu, J. Graphene-Metal Particle Nanocomposites. J. Phys. Chem. C 2008, 112, 19841-19845. (f) Krishnamurthy, S.; Kamat, P. V. Galvanic Exchange on Reduced Graphene Oxide: Designing a Multifunctional Two-Dimensional Catalyst Assembly. J. Phys. Chem. C 2013, 117, 571-577.

(7) Baek, S.; Kim, K. H.; Kim, M. J.; Kim, J. J. Morphology control of noble metal catalysts from planar to dendritic shapes by galvanic displacement. Appl. Catal., B 2017, 217, 313-321. 
(8) Porter, L. A., Jr.; Choi, H. C.; Ribbe, A. E.; Buriak, J. M. Controlled Electroless Deposition of Noble Metal Nanoparticle Films on Germanium Surfaces. Nano Lett. 2002, 2, 1067-1071.

(9) Choi, H. C.; Shim, M.; Bangsaruntip, S.; Dai, H. Spontaneous reduction of metal ions on the sidewalls of carbon nanotubes. J. Am. Chem. Soc. 2002, 124, 9058-9059.

(10) Vanysek, P. CRC Handbook of Chemistry and Physics, 92nd ed.; CRC Press, 2011; pp 80-89.

(11) Guo, R. H.; Jiang, S. X.; Yuen, C. W. M.; Ng, M. C. F.; Lan, J. W.; Yeung, Y. L.; Lin, S. J. Effects of Deposition Parameters of Electroless Copper Plating on Polyester Fabric. Fibers Polym. 2013, 14, 752-758.

(12) (a) Scott Bunch, J.; Verbridge, S. S.; Alden, J. S.; van der Zande, A. M.; Parpia, J. M.; Craighead, H. G.; McEuen, P. L. Impermeable Atomic Membranes from Graphene Sheets. Nano Lett. 2008, 8, 2458-2462. (b) Song, N.; Gao, X.; Ma, Z.; Wang, X.; Wei, Y.; Gao, C. A review of graphene-based separation membrane: Materials, characteristics, preparation and applications. Desalination 2018, 437, 59-72. 